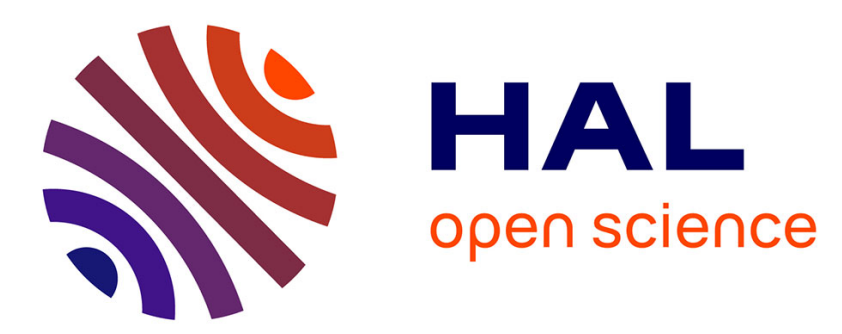

\title{
Image Similarity based Data Reduction Technique in Wireless Video Sensor Networks for Smart Agriculture Christian Salim, Nathalie Mitton
}

\section{To cite this version:}

Christian Salim, Nathalie Mitton. Image Similarity based Data Reduction Technique in Wireless Video Sensor Networks for Smart Agriculture. AINA 2021 - 35th International Conference on Advanced Information Networking and Applications, May 2021, Toronto, Canada. hal-03145329

\author{
HAL Id: hal-03145329 \\ https://hal.inria.fr/hal-03145329
}

Submitted on 18 Feb 2021

HAL is a multi-disciplinary open access archive for the deposit and dissemination of scientific research documents, whether they are published or not. The documents may come from teaching and research institutions in France or abroad, or from public or private research centers.
L'archive ouverte pluridisciplinaire HAL, est destinée au dépôt et à la diffusion de documents scientifiques de niveau recherche, publiés ou non, émanant des établissements d'enseignement et de recherche français ou étrangers, des laboratoires publics ou privés. 


\title{
Chapter 1 \\ Image Similarity based Data Reduction Technique in Wireless Video Sensor Networks for Smart Agriculture
}

\author{
Christian Salim and Nathalie Mitton
}

\begin{abstract}
Nowadays, to improve animal well being in livestock farming or beekeeping application, a wireless video sensor network (WVSN) can be deployed to early detect injury or Asiatic hornets attacks. WVSN represents a low-cost monitoring solution compared to other technologies such as the closed circuit television technology (CCTV). WVSNs are composed of low-power resource-constrained video sensor nodes (motes). These nodes capture frames from videos at a given frequency (frame rate) and wirelessly send them to the sink. The big amount of data transferred from the nodes to the sink consumes a lot of energy on the sensor node, which represents a major challenge for energy-limited nodes. In this paper, we introduce two complementary mechanisms to reduce the overall number of frames sent to the sink. First, the Transmission Data Reduction algorithm (TDR) run on the sensor node leverages the similarity degree of consecutive images. Second, the Inter-Nodes Similarity algorithm (INS) exploits the spatio-temporal correlation between neighbouring nodes in order reduce the number of captured frames. The results show a $95 \%$ data reduction, surpassing other techniques in the literature by $30 \%$ at least.
\end{abstract}

Key words: Wireless video sensor networks; Data Reduction; Geometric Correlation; Inter-Nodes Similarity.

\subsection{Introduction}

In recent years, the need for smart agriculture has increased [14]. Surveillance systems are deployed to support it, especially in remote sites in order for instance to monitor the animals behaviour, understand and protect the livestock and vegetables farming. To achieve it, a wireless video sensor network (WVSN) can serve as an

Christian Salim and Nathalie Mitton

Inria, France, e-mail: firstname. lastnamedinria.fr 
energy-efficient and low cost solution. In WVSN, the system normally operates periodically, sending all the captured frames to the sink [12]. However, the system can be an event driven system sending only the frames that show a change in the area of interest. The wireless video sensor nodes are energy-limited nodes that monitor an area of interest according to their Field of Views (FOVs) and send the videos/frames to the sink. Shooting a video and capturing its frames consume a lot of energy but sending this great number of frames to the sink is even worse. In order to cover all the area of interest and prevent black holes, in general, WVSN are such that several FoVs overlap with each other. To reduce the energy consumption at the sensor node level, we aim to reduce the amount of data sent to the sink leveraging the overlap between FoVs and by applying a node embedded lightweight algorithm in order to cope with the nodes limited processing and energy resources.

In this paper, we propose a two-level data reduction approach. The first one, Transmission Data Reduction algorithm (TDR), runs on each node and selects only the critical frames to be sent to the sink. The second one, the Inter-Nodes Similarity algorithm (INS), leverages the spatio-temporal correlation between neighbour nodes to reduce the number of sent and captured frames. The TDR algorithm compares consecutive captured frames on the node based on a norm simple euclidean distance similarity method [11]. According to the degree of similarity with the last frame sent to the sink, the node decides whether to send this frame or not [12]. If the decision is to send the image, the node creates a new image $i m g_{d i f f}$ that only includes the difference between the two compared images. $i m g_{d i f f}$ is sent to the sink instead of the whole image. In this case, data reduction is attained when several consecutive frames represent a high similarity percentage (stable situation). To furthermore increase the data reduction, the INS algorithm computes the correlated degree of neighbouring nodes and compares their frames using the same norm simple euclidean distance metric. If the result detects a similarity for $n$ consecutive frames, the node with the highest energy level sends the frames to the sink, the other one goes to idle mode and its frame rate is set to the minimum $F R=1$. We compare this new method to another algorithm from the literature based on the colour and edge similarities and on the number of consecutive frames sent per period [10]. The results show that our approach reduces the amount of energy by $95 \%$ outperforming the other approach, while capturing all the events.

The remainder of this paper is organised as follows. Section 1.2, browses the related work. Section 1.3 describes the transmission data reduction algorithm while Section 1.4 details the INS algorithm The experimental results and the comparison with other methods are given in Section 1.5. Finally, Section 1.6 concludes and provides perspectives and future work.

\subsection{Related work}

In this section, we discuss some previous works regarding data reduction in WSN and in WVSN in particular. A lot of techniques based on scheduling [6],[3], in- 
trusion location and clustering [13, 4], compressive sensing [16], data similarity [10], [11], and frame rate adaptation [15, 7] exist for data reduction and for energyefficiency. The authors in [13] used the kinematics functions to predict the next location of the intrusion in the area of interest in order to increase the frame rate adaptation of the targeted nodes. This approach reduced the energy dissipation of the overall network, since only the targeted nodes are capturing and not all the nodes in the zone of interest. This approach can come as a complementary solution to our method which focuses on the nodes detecting the intrusion and not on the projected positions of the intrusion. In [11] and [15], the authors worked on data reduction by adapting the frame rate of the nodes. This type of approaches is helpful and could be studied later on as a complementary work of the approach presented in this paper. The geometric correlation between several sensor nodes has been exploited by several authors such as $[9,1,8]$. In [1], the authors transform the coordinates of every point from 3D to a $2 \mathrm{D}$ coordinate system according to some reference points. Computing the disparity and the lengths of the reference vectors can determine whether for example two cameras are geometrically correlated according to a predefined threshold. In [10], the authors proposed a similarity function to detect the percentage of similarity between consecutive frames to attain data reduction by sending the critical frames to the sink. They merge it with a correlation overlapping technique at the coordinator level. The proposed colour-edge similarity function is time and energy consuming especially in the colour part where every channel is taken into consideration. They compared the frames on the coordinator level to further reduce the amount of frames. In this paper, the proposed method reduces the number of frames sent from each node to the coordinator by using the L2-norm distance to measure the similarity. It is a way less heavy to be implemented at the sensor node unlike other techniques that use the colour-edge and pixel similarities as in [10],[15],[7]. As explained in section 1.3 it is a less complex technique regarding the execution time comparison with other approaches for the same purpose of data reduction. Unlike other approaches that use a $3 \mathrm{D}$ to $2 \mathrm{D}$ transformation to implement a similarity algorithm as in [1], we complement the approach by proposing a 2-D spatial correlation between neighbour nodes based on some geometrical conditions using simple equations. If 2 nodes are said "correlated", the Inter-Nodes Similarity algorithm (INS) runs on both nodes, the one with the most residual energy sends the data, the other goes into idle transmission mode and lowers its frame rate to its minimum $F R=1$.

\subsection{Data Reduction}

In WSN, sensor-nodes operate periodically, and send a huge amount of data to the coordinator simultaneously. This scenario consumes a lot of energy on each sensor node representing the most important challenge in WSN in general. In WVSN, packets sent are multimedia, which emphasises even more the challenge of energy consumption. To overcome this challenge and to lower its importance on the fea- 
sibility of the network deployment, we proposed a data reduction method at the sensor node to reduce the number of raw images sent from each node to the sink. This technique combines two mechanisms presented by the Transmission Data Reduction algorithm (TDR) and the Inter-Nodes Similarity algorithm (INS).

\subsubsection{Transmission Data Reduction}

The Transmission Data Reduction algorithm (TDR) is described in Algo. 1. TDR algorithm leverages the consecutive frames similarity as in [11], using the norm simple euclidean distance similarity method to compare images. If two consecutive sensed frames are estimated as similar (no new information is represented in the second frame), the node does not send the second frame to the coordinator. The node counts the number of consecutive similar frames, if it surpasses $n b$ (the required number of consecutive similar frames), the state of the area of interest monitored by the node is considered as stable (situation=1). This technique uses the norm L2 relative error function tested in $\mathrm{C}++$ in OpenCV and compared to other techniques in [11] where the time to execute this technique is way less important than other techniques (edge, colour, ...) for the same purpose of data reduction. To apply this function, each image is transformed into a matrix. If the similarity norm $_{\text {sim }}=\operatorname{dist}\left(f r_{i}, f r_{\text {last }}\right)$ between those images does not surpass a certain threshold of similarity $\left(\right.$ norm $\left._{\text {sim }}<t h_{\text {sim }}\right)$, it means the images are not similar. In this case, the sensor-node creates and sends to the sink a new image called $i m g_{\text {diff }}$ by adopting the absolute images difference function as shown in the equation below:

$$
i m g_{\text {diff }}=\operatorname{absdiff}(M A T A, M A T B)
$$

This function computes the absolute value of the difference for each pixel, for each channel of the two matrices. The $i m g_{\text {diff } f}$ is an image that represents the difference between both images using less bits. In this case, the size in Bytes of the transmitted packets decreases because only the difference between images is sent rather than the full new image.

An example of the $i m g_{d i f f}$ is shown in Figure 1.2c representing the difference image $i m g_{\text {diff }}$ of the frames presented in Figures 1.2a and 1.2b taken from the dataset in $[5]^{1}$. As we can see, the shadow of the moving person and the person are the only differences taken into account. The last sent image to the coordinator is stored on the sensor node. Every new frame is compared to this last frame sent to the sink, it can replace this last frame if it represents a critical event or a change in the monitored zone as shown in Algo 1. In the remainder of this paper, the sent image $i m g_{d i f f}$ is called critical frame. We called it critical, because the node sends only an image to the coordinator if an event or a change is happening in the area of interest. This whole process is explained in Fig. 1.1. Note that, the reconstruction algorithm at the sink level is out of the scope of this paper.

\footnotetext{
${ }^{1}$ http://changedetection.net
} 


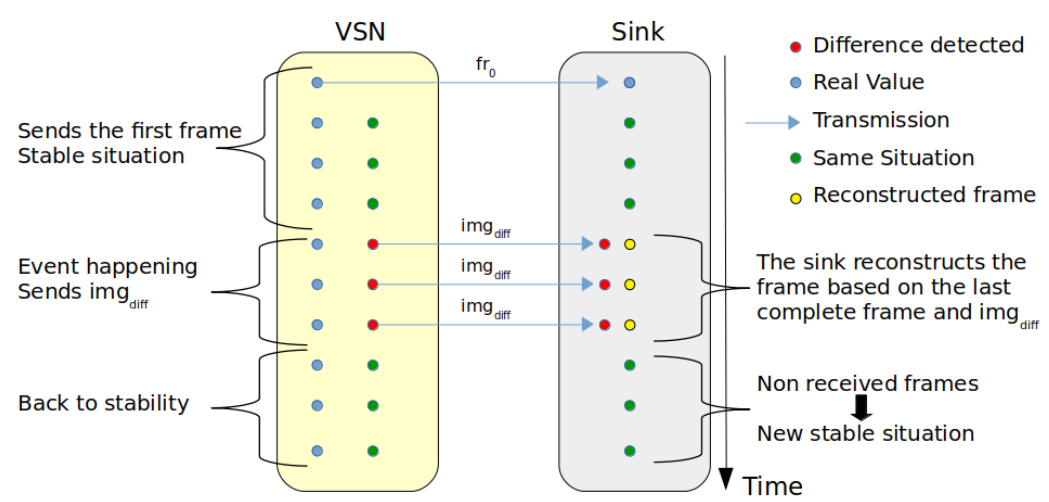

Fig. 1.1: Video sensor node and sink behaviours

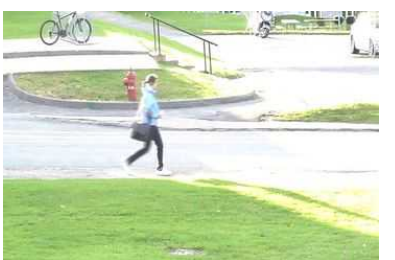

(a) The First Frame

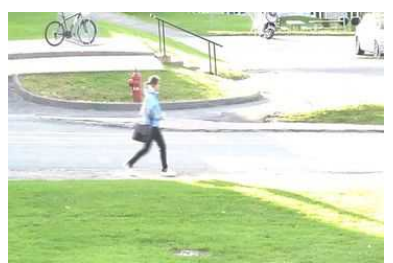

(b) The Second Frame

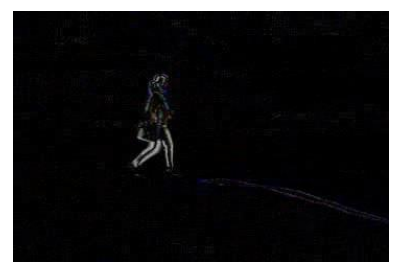

(c) The Difference Frame

Fig. 1.2: The Difference Scenario

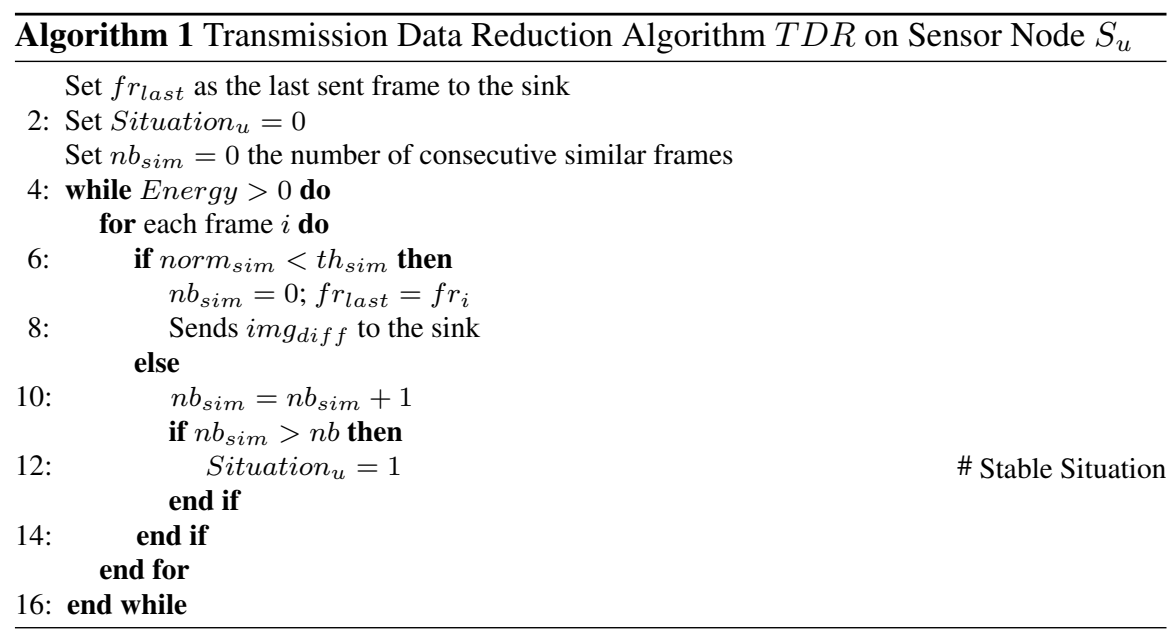

\subsection{Inter-Nodes Correlation: The Overlapping Method}

In the above sections, we were interested in reducing the amount of sent frames from each node to the sink by applying a similarity function at the sensor node itself. To 


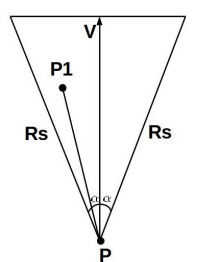

(a) Field of View

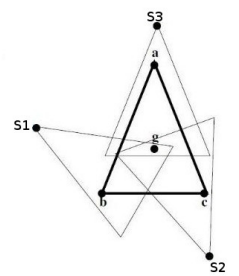

(b) Two points Method

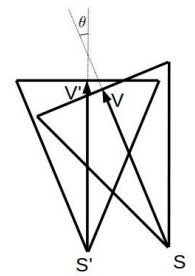

(c) Angle Condition

Fig. 1.3: Overlapping Model

furthermore reduce the data transmission, we now focus on the spatial correlation between neighbouring sensor nodes. In our approach, two nodes are considered as neighbours if they are in the communication range of each other. Neighbour nodes exchange their vector of metrics defined in the video sensing model below to check whether their FoVs overlap enough to apply the learning process and the similarity function. We apply the same norm distance similarity function on frames captured by two overlapping neighbour sensor nodes. This technique reduces the number of the overall captured and sent frames from the nodes to the sink, by only sending one out of the two frames, depending on the residual energy of the two nodes as shown in Algorithm 2. Before describing the Inter-Nodes Correlation (INS) approach, we introduce the video sensing model, the characteristics of every video sensor node and the geometric condition to determine what nodes overlap and can thus proceed with the INS algorithm.

\subsubsection{Video Sensing Model}

We consider a 2-D model of a video sensor node where $z=0$ (XOY plane) and all the captured frames are compared as 2-D images not taking account of the third dimension. A video sensor node $S$ is represented by the Field of View (FoV) of its camera. Unlike a wireless sensor which range is omnidirectional, a FoV covers only a part of the surrounding area of a video sensor as shown in Fig. 1.3a. A FoV is a vector of 4-tuple $S\left(P, R_{s}, \vec{V}, \alpha\right)$ where $P$ is the position of $S, R_{s}$ is its sensing range, $\vec{V}$ is the vector representing the line of sight of the camera's FoV e.g. its sensing direction, and $\alpha$ is the offset angle of the FoV.

In [10] the authors presented the FoV with 4 points a,b,c and the gravity centre $\mathrm{g}$ as shown in Fig. 1.3b. The overlapping area is detected based on those points as explained in the upcoming subsections. A point $P_{1}$ is said to lay in the FoV of a video sensor node $S$ at position $P$ if and only if the two following conditions are satisfied: 
1. $d\left(P, P_{1}\right) \leq R_{s}$, where $d\left(P, P_{1}\right)$ is the Euclidean distance between $P$ and $P_{1}$ : $\left\|\overrightarrow{P P_{1}}\right\| \leq R_{s}$

2. The angle between $\overrightarrow{P P_{1}}$ and $\vec{V}$ is within $[-\alpha,+\alpha]: \overrightarrow{P P_{1}} \cdot \vec{V} \geq\left\|\overrightarrow{P P_{1}}\right\| \times$ $\|\vec{V}\| \times \cos (\alpha)$.

In the remainder of this paper, we consider that all video nodes are identical, with the same sensing range $R_{s}$ and the same offset angle $\alpha$. In the next section, we discuss the spatial correlation of overlapping neighbour nodes based on some geometrical conditions, and how we use it in order to increase the data reduction at the sensor node level.

\subsubsection{Overlapping Sensor Nodes}

In this part, we describe the geometric condition which determines if two neighbours are overlapping nodes. This condition is a combination of the angle condition between the FoV's of the nodes and the ratio of the overlapping area between them.

\subsubsection{The Angle Condition}

The angle between two neighbouring sensor nodes is defined as the angle between the vectors of their FoVs. If the angle between the FoVs of two neighbouring nodes is wide, these two nodes can not take part in the similarity comparison function to decide which one among them sends the data to the sink. Indeed, that means that they are not sensing the same area of interest. A frame from two different perspectives can be widely different. To be able to define two neighbour sensor nodes as overlapping sensor nodes, the angle between their FoVs must not surpass a certain angle threshold. In order to determine the angle between vectors $\left(\vec{V}\right.$ and $\left.\vec{V}^{\prime}\right)$ of the sensor nodes $S$ and $S^{\prime}$ respectively as shown in Figure 1.3c, we use the scalar product between those sensor nodes. Since all sensor nodes are identical, both vectors $\vec{V}$ and $\vec{V}^{\prime}$ have the same length $l$. The scalar product can be calculated in two formats. The first one according to their coordinates (x and y) where $\vec{V}=\left(X_{V}, Y_{V}\right)$ and $\vec{V}^{\prime}=$ $\left(X_{V^{\prime}}, Y_{V^{\prime}}\right)$ :

$$
\vec{V} \cdot \overrightarrow{V^{\prime}}=X_{V} \times X_{V^{\prime}}+Y_{V} \times Y_{V^{\prime}}
$$

The second format is given according to the length of each vector and to the angle between both, as follows:

$$
\vec{V} \cdot \vec{V}^{\prime}=l^{2} \times \cos \left(\vec{V}, \vec{V}^{\prime}\right)
$$

Where $l=\left\|\overrightarrow{V^{\prime}}\right\|=\|\vec{V}\|$.

The below equation defines and computes the angle $\theta$ between the two vectors according to both formats of the scalar product: 


$$
\theta=\arccos \left(\left(X_{V} \times X_{V^{\prime}}+Y_{V} \times Y_{V^{\prime}}\right) / l^{2}\right)
$$

If an angle threshold thangle is defined as 30 degrees, the angle between $\vec{V}$ and $\vec{V}^{\prime}$ must remain less than 30 degrees so the two sensor nodes $S$ and $S^{\prime}$ can proceed to the next step (the two points strategy), to be able at the end to take part in the similarity function process.

\subsubsection{The two Points Condition}

Inspired from [10] we present below the two points condition as the second part of the geometric condition. A node $S^{\prime}$ satisfies the two points condition with another node $S$ if $g$ (the gravity centre of abc) belongs to the FOV of $S$ alongside any other point between a, b and c from $S^{\prime}$ s FOV as shown in Figure 1.3b. In this figure, $S_{1}, S_{2}$ and $S_{3}$ satisfy this condition separately with $S$. In this scenario, each sensor node can be a candidate alongside $S$ to apply the learning process and the similarity function between them on the one having the most residual energy. However, only $S_{1}$ and $S_{2}$ satisfy at the same time the angle and the two points conditions. For this purpose, $S_{1}$ and $S_{2}$ will be the nodes selected as two overlapping sensor nodes to test the inter nodes similarity algorithm $I N S$ as explained in the next section.

\subsubsection{Inter-Nodes Similarity}

As mentioned earlier, the comparison between the frames captured by overlapping sensor nodes is done at the start of each stable situation in the zone of interest. We used the same norm simple euclidean distance function to measure the similarity. If the similarity computed ratio $I N_{s i m}$ is greater than a predefined threshold $I N_{t h}$ for the first frame as shown in Algo. 2, it means that the two correlated nodes present the same information when shooting the zone of interest. In this case, only one

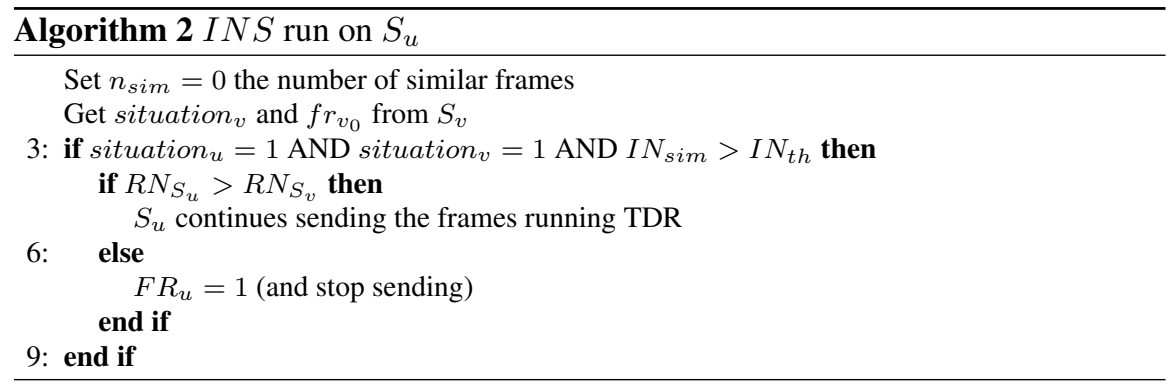

out of the two sensor nodes is selected to send the data, depending on the residual energy $R N$ of both nodes. If $S_{u}$ has more energy left than $S_{v}\left(R N_{S_{u}}>R N_{S_{v}}\right)$, 
$S_{u}$ continues to send the data while $S_{v}$ goes to transmission idle mode and its frame rate is reduced to $F R=1$ frame per second on $S_{v}$. Once an event occurs in the FoV of $S_{v}$ the frame rate is back to its maximum of 30 frames per second. Fig 1.4 shows

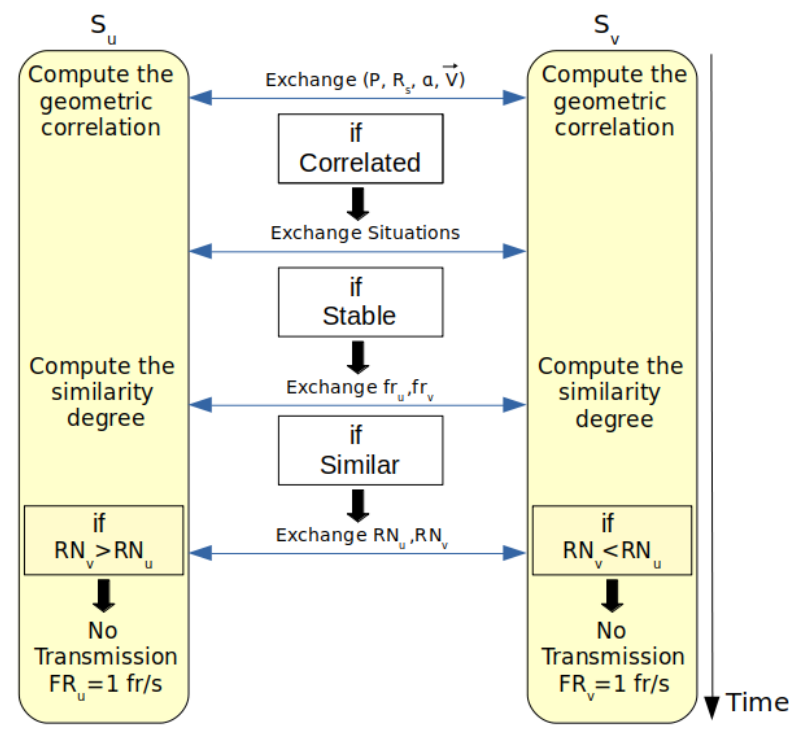

Fig. 1.4: Two overlapping sensor nodes behaviour running INS

the behaviour of two neighbour nodes while applying this overlapping technique and INS algorithm.

\subsection{Experimental Results}

Several simulations have been conducted to validate our approach at the sensor node, aiming to minimise the number of frames sent from the node to the sink. We compare our approach to MASRA and SSA algorithms in [10] and PPSS algorithm in [2]. We have used a Matlab simulator. A scenario is introduced in Figure $1.3 \mathrm{c}$ where 2 video-sensor nodes $\mathrm{S} 1, \mathrm{~S} 2$ are deployed as overlapped neighbour nodes to monitor the same area of interest from different perspectives as shown in Figure $1.3 \mathrm{c}$. The angle threshold is set to 15 degrees, however in this case $\alpha=10$ and $S_{1}$ covers the gravity centre and one other point of the FoV of $S_{2}$, which makes them two overlapping neighbour nodes in order to apply the INS algorithm on both. The main purpose in our work is to send to the sink the frames that represent the critical situations while maintaining the information by applying TDR and INS algorithms. We have used 2 Microsoft LifeCam VX-800 cameras to shoot a short video of 120s 


\begin{tabular}{cccc}
\hline \multicolumn{3}{c}{ Node } & \multicolumn{3}{c}{ Situation } & Captured Frames Sent Frames \\
\hline$S_{1}$ & Stable & 2300 & 2 \\
$S_{1}$ & Event & 1200 & 240 \\
$S_{2}$ & Stable & 2300 & 2 \\
$S_{2}$ & Event & 1200 & 260 \\
$A L L$ & All & 7200 & 504
\end{tabular}

Table 1.1: TDR algorithm over 120s

\begin{tabular}{ccc}
\hline Total Frames & Type Frames & Size Frames \\
\hline 7200 & Captured all & $216000 \mathrm{~KB}$ \\
504 & Critical all & $15120 \mathrm{~KB}$ \\
504 & $i m g_{\text {diff }}$ & $10080 \mathrm{~KB}$ \\
\hline
\end{tabular}

Table 1.2: Overall data and size reduction

(3600 frames captured per node), each camera is connected to a laptop to do the processing via a Matlab simulator. Each camera captures 30 frames/s. In our scenario, an intrusion is detected at the following time-intervals:

$S 1$ : 40 s from 15 to $55 ; \mathrm{S} 2: 40$ s from 20 to 60 .

In the rest of the time, both sensor nodes had a stable situation. After conducting several simulations, the number of high similarities needed to detect a stable situation is set to $n=60$ which means 2 seconds of stability. The similarity threshold $t h_{\text {sim }}$ is set to $85 \%$ in TDR algorithm and $75 \%$ in INS algorithm. Note that a full compressed image size is equal to $30 \mathrm{~KB}$ and the difference image $i m g_{\text {diff }}$ size is $20 K B$. The thresholds and all the parameters can be adapted according to the application and the QoS required.

\subsubsection{Transmission data reduction}

Table 1.1 shows the number of frames sent by each node to the sink over 120 seconds using the TDR algorithm. This algorithm allows the reduction of the number and size of frames transmitted by the sensor-node as shown in Tables 1.1 and 1.2.

A transmission data reduction of more than $90 \%$ is reached by sending only the critical frames. However the size reduction reached $95 \%$ due to the image difference $i m g_{\text {diff } f}$ applied on every selected/critical frame. Once in a stable situation, the first frame is sent and due to similarity all the other frames are not transmitted to the sink.

\subsubsection{Inter-Nodes Similarity}

The INS algorithm runs on both nodes at the same time with TDR algorithm. In this section we consider that $S_{1}$ has more residual energy than $S_{2}$, which puts $S_{2}$ into the idle mode and reduces its frame rate to $F R_{2}=1$ frame per second. In this case the data reduction is emphasised also on the sensing process for $S_{2}$ as shown in Table 1.3. In this case, the number of frames sent to the sink remains the same as in TDR alone, but the number of captured frames reduces by more than $50 \%$ on $S_{2}$ and $33 \%$ overall. 


\begin{tabular}{|c|c|c|c|}
\hline Node & Situation & Captured Fr & at Frames \\
\hline$S_{1}$ & Stable & 2300 & 2 \\
\hline$S_{1}$ & Event & 1200 & 240 \\
\hline$S_{2}$ & Stable & 133 & 2 \\
\hline$S_{2}$ & Event & 1200 & 260 \\
\hline$A L L$ & All & 4833 & 504 \\
\hline
\end{tabular}

Table 1.3: TDR and INS - 120 seconds

\begin{tabular}{cccc}
\hline Technique & Captured & Sent & Size in KB \\
\hline All data & 7200 & 7200 & 216000 \\
MASRA+ SSA[10] & 2540 & 1310 & 39300 \\
PPSS[2] & 3600 & 3600 & 108000 \\
TDR + INS & 4833 & 504 & 10080 \\
\hline
\end{tabular}

Table 1.4: S1 and S2 data reduction

\subsubsection{Comparison}

We compare to the scheduling algorithm PPSS [2] and to the MASRA algorithm [10] where the colour and edge similarities were both present to detect the difference between images. The difference between those approaches in terms of data reduction is shown in Table 1.4. As we can notice from Table 1.4, TDR and INS algorithms outperform MASRA algorithm [10] and PPSS algorithm [2] in terms of transmission data reduction by $70 \%$ and $90 \%$ respectively and in terms of the size of the data transmitted. This technique reduced the transmitted data by $95 \%$ and the sensed data by $33 \%$ of the overall data captured and transmitted. However, the sensing data reduction is better on MASRA algorithm by $45 \%$ which implements the frame rate adaptation of each node according to the scene but computes the geometric correlation on the sink not on the node, in this purpose they transmit 3 times more than our current approach. The overall gain remains positive by more than $30 \%$.

\subsection{Conclusion}

In this paper, we introduced a distributed data reduction approach for wireless video sensor network. This approach is composed of two complementary algorithms, the transmission reduction algorithm (TDR) and the inter-nodes similarity (INS) algorithm. TDR detects the similarities between consecutive captured frames and decides to send only the difference image of each critical frame to the sink. The results show that the proposed algorithms did not miss any event in the recorded video sequence. The second part of the approach consists in a geometrical condition computed by neighbour nodes to detect whether they overlap and reduce accordingly their frame rate or transmission rate. Comparing our approach with MASRA and PPSS algorithms in terms of data reduction shows that our algorithm outperforms both algorithms by $30 \%$ and $90 \%$ respectively. As future works, first of all we aim to compare the relevance of our mathematical model by comparing it to other approaches. In order to increase the data reduction, the frame rate adaptation technique at the sensor node level can be taken into account. 


\section{Acknowledgements}

This work was partially supported by a grant from CPER DATA, by LIRIMA Agrinet project and the Inria/CEFIPRA Associate team DC4SCM.

\section{References}

1. Dai, R., Akyildiz, I.F.: A spatial correlation model for visual information in wireless multimedia sensor networks. IEEE TRANSACTIONS ON MULTIMEDIA 11(6), 1148-1159 (2009)

2. Elson, J., Girod, L., Estrin, D.: Fine-grained network time synchronization using reference broadcasts. SIGOPS Operating System Rev. pp. 147-163 (2002)

3. Feng, J., Zhao, H.: Energy-balanced multisensory scheduling for target tracking in wireless sensor networks. Sensors 18(10), 3585 (2018)

4. Ghosal, A., Halder, S.: A survey on energy efficient intrusion detection in wireless sensor networks. Journal of Ambient Intelligence and Smart Environments 9, 239-261 (2017)

5. Goyette, N., Jodoin, P.M., Porikli, F., Konrad, J., Ishwar, P.: changedetection.net: A new change detection benchmark dataset. In: IEEE Workshop on Change Detection (CDW-2012) (2012)

6. Jiang, B., Ravindran, B., Cho, H.: Probability-based prediction and sleep scheduling for energy-efficient target tracking in sensor networks. IEEE TRANSACTIONS ON MOBILE COMPUTING 12(4) (2013)

7. Monika, R., Hemalatha, R., Radha, S.: Energy efficient surveillance system using wvsn with reweighted sampling in modified fast haar wavelet transform domain. Multimed Tools Appl 77 (2018)

8. Mowafi, M.Y., Awad, F.H., Aljoby, W.A.: A novel approach for extracting spatial correlation of visual information in heterogeneous wireless multimedia sensor networks. Computer Networks 71, 31-47 (2014)

9. Nu, T.T., Fujihashi, T., Watanabe, T.: Power-efficient video uploading for crowdsourced multiview video streaming. In: IEEE Global Communications Conference (GLOBECOM) (2018)

10. Salim, C., Makhoul, A., Couturier, R.: Similarity based image selection with frame rate adaptation and local event detection in wireless video sensor networks. Multimed Tools Appl 78, 5941-5967 (2019)

11. Salim, C., Makhoul, A., Couturier, R.: Energy-efficient secured data reduction technique using image difference function in wireless video sensor networks. Multimed Tools Appl 79, 18011819 (2020)

12. Salim, C., Makhoul, A., Darazi, R., Couturier, R.: Combining frame rate adaptation and similarity detection for video sensor nodes in wireless multimedia sensor networks. In: IWCMC (2016)

13. Salim, C., Makhoul, A., Darazi, R., Couturier, R.: Kinematics based approach for data reduction in wireless video sensor networks. In: Int. Conf. on Wireless and Mobile Computing, Networking and Communications (WiMob) (2018)

14. Salim, C., Mitton, N.: Machine learning based data reduction in wsn for smart agriculture. In: International Conference on Advanced Information Networking and Applications. pp. 127138. Springer (2020)

15. Tayeh, G.B., Makhoul, A., Demerjian, J., Laiymani, D.: A new autonomous data transmission reduction method for wireless sensors networks. In: IEEE Middle East and North Africa Communications Conference (MENACOMM) (2018)

16. Veeraputhiran, A., Sankararajan, R.: Feature based fall detection system for elders using compressed sensing in wvsn. Wireless Netw 25, 287-301 (2019) 